\title{
Research on Performance Evaluation of Construction project based Analytic Hierarchy Process and Fuzzy Comprehensive Evaluation
}

\author{
Jinming Liu \\ School of Management, Tianjin University of Technology, Tianjin 300384, China \\ liujinming20103227@163.com
}

\begin{abstract}
Performance evaluation which based on Analytic Hierarchy Process (AHP) and Fuzzy Comprehensive Evaluation is a progress to verify architecture engineering construction's efficiency and equity and economy. Under this method, according to fundamental principles of fuzzy mathematics and the evaluation criterion which we already have, the valuator who have to consider a variety of factors which can affect project performance should organize professors give each factor a score to analyze how about the architecture engineering construction. This paper discussed the basic principle and steps of the method and have evaluated the architecture engineering construction performance successfully. Therefore, this method provides a more accurate way of evaluation about architecture engineering construction Performance evaluation, which is helpful to reduce the influence of subject consciousness when it times to evaluate project performance. In addition, the paper gives the feasibility and limitations of this method in practical applications.
\end{abstract}

Keywords: Fuzzy comprehensive evaluation; Analytic Hierarchy Process; performance evaluation; architecture engineering construction.

\section{基于层次分析法与模糊综合评价的建设项目绩效评价研究}

\author{
刘进明 \\ 天津理工大学, 天津 中国
}

摘 要: 基于层次分析法与模糊综合评价法的建设项目绩效评价是在考虑影响项目绩效的多种 因素的基础上, 根据模糊数学的基本原理, 对各影响因素给出评价标准, 并在此基础上通过 专家打分进行建设项目绩效评价的过程。本文探讨了该方法的基本原理和步骤, 并运用此方 法成功地对建设项目绩效评价进行了评估, 为建设项目绩效评价提供了一种更为精确的评价 方式, 有利于减少主观意识对绩效评价结果的影响。此外, 本文给出了此方法实际应用中的 可行性和局限性。

关键词：模糊综合评价；层次分析法；建设项目；绩效评价

\section{1. 前言}

随着大型公共项目投资逐年递增, 财政部《财政支出绩效评价管理暂行办法》(财预 [2011]285 号）、《关于印发〈预算绩效评价共性指标体系框架〉的通知》（财预 [2013]53 号）等文件 相继出台, 这表明财政资金使用效率越来越受到财政主管部门的重视。但在实际工程实践中, 比较常用的绩效评价方法主要有逻辑框架法、成功度法、层次分析法、对比法等基本方法 ${ }^{[1]}$ 。 无一例外的是, 这些方法的局限性在于评价结果容易受到主观意识的影响。与此同时, 我国 建设项目逐年增多, 效评价管理水平不尽如人意, 基础数据有待完善, 各种评价方法都不能 很好地反应公共项目的绩效水平。因此, 研究基于层次分析法与模糊综合评估法的公共项目 绩效评价十分有必要。 


\section{2. 层次分析法的基本原理与步骤}

\section{1 层次分析法的基本原理}

层次分析法 (Analytic Hierarchy Process 简称 AHP) 作为一种简便灵活的定性与定量相结合的 多准则决策方法, 由美国著名运筹学家 T.L.Saaty 等人于 20 世纪 70 年代提出 ${ }^{[2]}$ 。其主要优点 是逻辑性、系统性、实用性较强, 缺点为受评价人主观意识影响较大, 评价结果的客观性存 在一定的差距。

2.2 层次分析法的步骤

第一，建立层次结构模型

以选择旅游景点为例, 层次结构模型如图 1 所示。

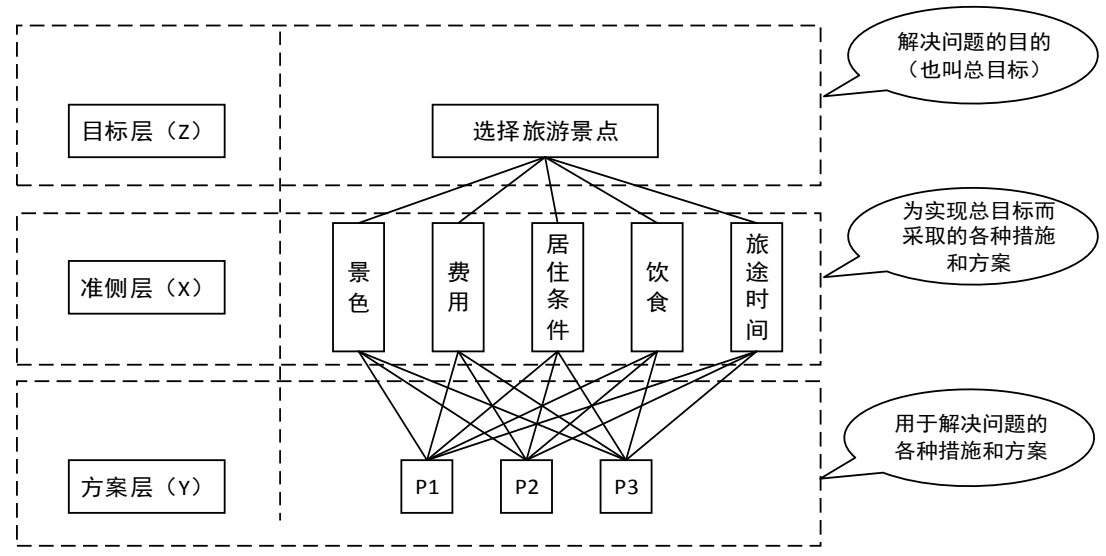

图1 层次结构模型

第二, 构造成对比较矩阵 (判断矩阵)

设某层有 $\mathrm{n}$ 个因素, 即 $\mathrm{X}=\left\{\mathrm{x}_{1}, \mathrm{x}_{2}, \mathrm{x}_{3}, \mathrm{x}_{4}, \cdots \cdots \mathrm{x}_{\mathrm{n}}\right\}$, 将这 $\mathrm{n}$ 个元素两两比较, 比较方式为从 $\mathrm{x}_{1}, \mathrm{x}_{2}, \ldots, \mathrm{x}_{\mathrm{n}}$ 中任取 $\mathrm{xi}_{\mathrm{i}}$ 与 $\mathrm{x}_{\mathrm{j}}$, 比较他们对于他们上一层的因素 $\mathrm{Z}$ 的贡献(或重要性)大小 ${ }^{[3]}$. 按照如下” $1-9$ 比例 尺度”给 $\mathrm{x}_{\mathrm{i}} / \mathrm{x}_{\mathrm{j}}$ 赋值.如表 1 所示。

表1 1-9比例尺度

\begin{tabular}{|c|c|}
\hline 尺度 $\mathrm{X}_{\mathrm{ij}}$ & 含义 \\
\hline 1 & $\mathrm{xi}$ 与 $\mathrm{xj}$ 的影响相同 \\
\hline 3 & $\mathrm{xi}$ 与 $\mathrm{xj}$ 的影响稍强 \\
\hline 5 & $\mathrm{xi}$ 与 $\mathrm{xj}$ 的影响强 \\
\hline 7 & $\mathrm{xi}$ 与 $\mathrm{xj}$ 的影响明显地强 \\
\hline 9 & $\mathrm{xi}$ 与 $\mathrm{xj}$ 的影响绝对地强 \\
\hline $2,4,6,8$ & $\mathrm{xi}$ 与 $\mathrm{xj}$ 的影响之比在上述两个相邻等级之间 \\
\hline $1,1 / 2, \cdots . .1 / 9$ & $\mathrm{xi}$ 与 $\mathrm{xj}$ 的影响之比为上面 $\mathrm{aij}$ 的互反数 \\
\hline
\end{tabular}

在 1-9 比例尺度的基础上, 得到判断矩阵为 $A=\left(X_{i j}\right), X_{i j}>0, X_{j i}=1 / X_{i j}$, 继续以假日旅游为例, 判断矩阵 $A=\left[\begin{array}{ccccc}1 & 2 & 7 & 5 & 5 \\ 1 / 2 & 1 & 4 & 3 & 3 \\ 1 / 7 & 1 / 4 & 1 & 1 / 2 & 1 / 3 \\ 1 / 5 & 1 / 3 & 2 & 1 & 1 \\ 1 / 5 & 1 / 3 & 3 & 1 & 1\end{array}\right]$ 其中, $X_{12}=1 / 2$ 表示 $x_{1}$ 与 $x_{2}$ 对旅游地选择这个目标的重要性 之比为 $1: 2$, 即认为费用更重要。

第三，计算权向量并进行一致性检验

实际决策问题中, 通常把某一变量 $\mathrm{M}$ 变成变量 $\mathrm{x}_{1}, \mathrm{x}_{2}, \mathrm{x}_{3}, \mathrm{x}_{4}, \cdots \cdots \mathrm{x}_{\mathrm{n}}$ 的组合, 及 $\mathrm{M}=\mathrm{W}_{1} \mathrm{X}_{1}+\mathrm{W}_{2} \mathrm{X}_{2}+\ldots \mathrm{W}_{\mathrm{n}} \mathrm{X}_{\mathrm{n}}$ 其中, $w i>0, \sum_{i=1}^{n} w_{i}=1$, 则 $\mathrm{w}_{1}, \mathrm{w}_{2}, \ldots . ., \mathrm{w}_{\mathrm{n}}$ 叫做各因素对目标 $\mathrm{M}$ 的 权重, $W=\left(w_{1}, w_{2}, \cdots w_{n}\right)^{T}$ 叫做权向量。 

$\mathrm{a}$.将 $\mathrm{A}$ 的每一列向量归一化得 $w_{i j}=a_{i j} / \sum_{j=1}^{n} a_{i j}$
b. 对 $\tilde{w}_{i j}$ 按行求和得 $w_{i}=\frac{w_{i}}{\sum_{j=1}^{n} W_{i}}$

c. 将 $w_{i}$ 归一化为 $w_{i}=\frac{w_{i}}{\sum_{j=1}^{n} w_{i}}, W=\left(w_{1}, w_{2}, \cdots w_{n}\right)^{T}$ 即为近似特征根（权向量）

d. 计算 $\lambda=\frac{1}{n} \sum_{i=1}^{n} \frac{(A w)_{i}}{w_{i}}$, 作为最大特征根的近似值。

则一致性指标 $C I=\frac{\lambda-n}{n-1}$ 。其中 $\mathrm{CI}=0$ 时 $\mathrm{A}$ 一致, $\mathrm{CI}$ 越大, 表示 $\mathrm{A}$ 的不一致程度越严重。 随机一致性指标RI如表2所示。

表2 随机一致性指标RI

\begin{tabular}{|c|c|c|c|c|c|c|c|c|c|c|c|}
\hline $\mathrm{n}$ & 1 & 2 & 3 & 4 & 5 & 6 & 7 & 8 & 9 & 10 & 11 \\
\hline $\mathrm{RI}$ & 0 & 0 & 0.58 & 0.90 & 1.12 & 0.24 & 0.32 & 1.41 & 1.45 & 1.49 & 1.51 \\
\hline
\end{tabular}

在已知 $\mathrm{C}$ 和I 的基础上, 一致性比率 $\mathrm{CR}=\mathrm{CI} / \mathrm{RI}$, 当 $\mathrm{CR}<0.1$ 时, $\mathrm{A}$ 的不一致性程度在容许范 围内,此时可用 $\mathrm{A}$ 的特征向量作为权向量。

第四, 分别计算各层次元素的组合权重

所谓组合权重, 指的是最低层次目标相对于总目标的相对权重, 即把第三部结果进行分析整 合进而得出结果。需要注意的是, 计算各层次元素的组合权重必须从上到底依次进行。

最终, AHP的结果是方案层中各决策目标对决策方案的优先权重顺序, 并在此基础上进行下 一步的决策。

\section{3. 模糊综合评估法的基本原理与数学模型}

3.1 模糊综合评估法的基本原理

提到模糊综合评估法, 就不得不提到模糊数学。 20 世纪 60 年代中期, 美国电机工程与计算机 科学系教授 L.A.Zadeh (扎德) 发表文章《模糊集》，从而宣告模糊数学的诞生 ${ }^{[4]}$ 。而模糊综 合评价则是以模糊数学为基础的综合评价方法。该方法以隶属度理论为起点, 将定性评价转 化为定量评价, 即用模糊数学对由多种因素制约的对象或者事物做出总体的评价。此方法的 主要特点是考虑到多重因素的基础上不再给出“非黑即白”的评价结论, 而是以一个模糊集合 的形式表示对事物的最终评价。

3.2 模糊综合评估法的数学模型

第一，确定评价对象的因素集

对由多因素影响的某一事物进行评价, 将评价指标细分为 $\mathrm{m}$ 个, 分别记为 $\mathrm{u}_{1}, \mathrm{u}_{2}, \ldots . . . \mathrm{u}_{\mathrm{m}}$, 这些 评价因素即为评价某事物的有限集合 $U=\left\{\mathrm{u}_{1}, \mathrm{u}_{2}, \ldots . . . \mathrm{u}_{\mathrm{m}}\right\}$

第二, 确定评价对象的评语级

根据不同的事物评价的需要, 将事物的评语分成 $\mathrm{n}$ 个等级, 分别记为 $\mathrm{v}_{1}, \mathrm{v}_{2}, \ldots . . . \mathrm{V} \mathrm{n}$, 这些评价等 级为评价某事物的好坏有限集合 $\mathrm{V}=\left\{\mathrm{V}_{1}, \mathrm{~V}_{2}, \ldots . . . \mathrm{V}_{\mathrm{n}}\right\}$

其中, : $V_{j}$ 代表第 $j$ 个评价结果, $j=1,2, \ldots, n . n$ 为总的评价结果数，一般划分为 $3-5$ 个等级。

第三，确定评价因素的权重向量

评价事物的实际过程中, 各评价因素对事物的重要性程度显然是不一致的。以此为出发点, 评价因素权重集合实际上是一个因素集合 $U$ 这一论域上的一个模糊集合 $A$, 自然也是一个有 限集, 即因素权重集合也同因素集与评语集一样，是有限模糊集合。其中 $A=\left(a_{1}, a_{2}, \ldots . . ., a_{m}\right)$ 其论域为 $\mathrm{U}, \mathrm{a}$ 是 $\mathrm{A}$ 中相应元素的隶属度，且 $a_{i} \geq 0 ; \sum a_{i}=1$

第四, 构建模糊关系矩阵 $\mathrm{R}$ 
依次对每个评价因素 $u_{i}$ 进行量化, 也就是确定评价对象对各等级模糊子集的隶属度, 进而得 到模糊关系矩阵 $R=\left(\begin{array}{cccc}r_{11} & r_{12} & \cdots & r_{1 n} \\ r_{21} & r_{22} & \cdots & r_{2 n} \\ \cdots & \cdots & \cdots & \cdots \\ r_{m 1} & r_{m 2} & \cdots & r_{m n}\end{array}\right)$

第五，构建模糊综合评价的数学模型

在得出权重向量 $\mathrm{A}$ 和模糊关系矩阵 $\mathrm{R}$ 的基础上，模糊综合评价的数学模型为

$$
B=A \circ R=\left(\begin{array}{llll}
a_{1} & a_{2} & \cdots & a_{m}
\end{array}\right)\left(\begin{array}{cccc}
r_{11} & r_{12} & \cdots & r_{1 n} \\
r_{21} & r_{22} & \cdots & r_{2 n} \\
\cdots & \cdots & \cdots & \cdots \\
r_{m 1} & r_{m 2} & \cdots & r_{m n}
\end{array}\right)=\left(\begin{array}{llll}
b_{1} & b_{2} & \cdots & b_{m}
\end{array}\right)
$$

其中, $b_{j}$ 表示被评级对象从整体上看对评价等级模糊子集元素 $v_{j}$ 的隶属程度

将 $B$ 进行归一化处理后得到 $B_{1}$ ，然后将 $B_{1}$ 与有限模糊评语集 $A$ 作乘，即 $G=B_{1} \circ V^{T}$ 。 最终, 模糊综合评价的结果是取所有评价中的中位数作为本次评价的最终结论, 从一定意义 上消减了因主观意识导致的评价偏差 ${ }^{[5]}$ 。

\section{4. 运用层次分析法与模糊综合评估法进行公共项目绩效评价}

\section{1 建立指标体系}

根据财政部《关于印发〈预算绩效评价共性指标体系框架〉的通知》（财预[2013]53 号）文 件中的项目支出绩效评价共性指标体系框架，以财政资金投资的建设项目为例，建立建设项 目绩效评价指标体系的模糊综合评价三层模型如图 2 所示。

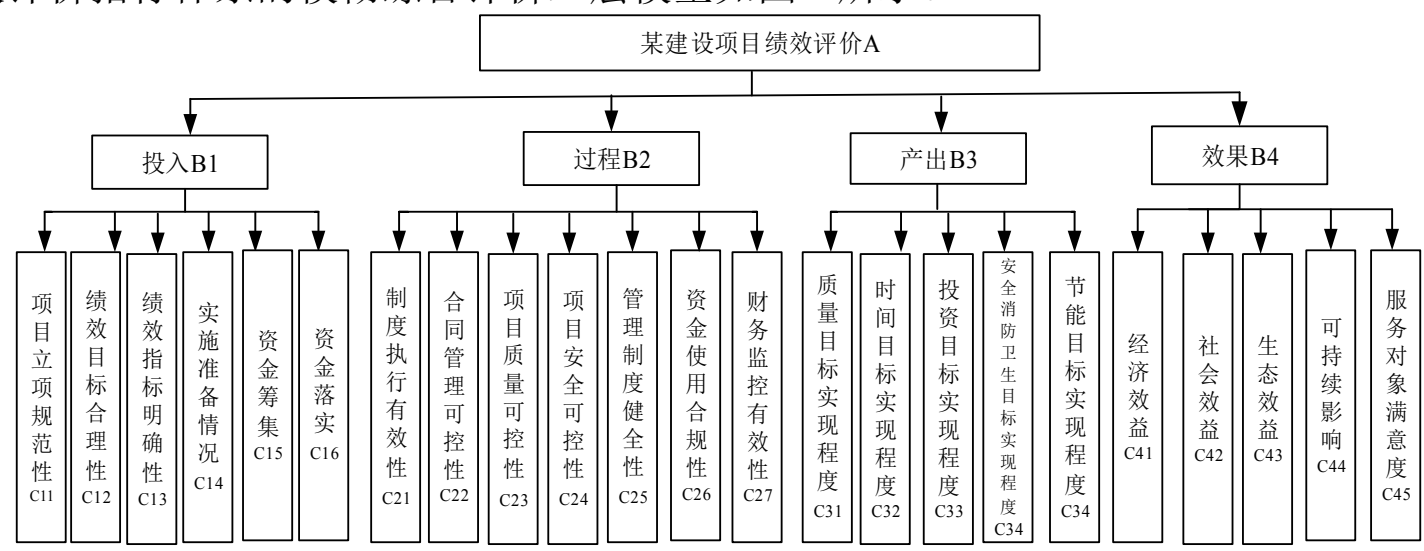

\section{图2 某建设项目综合绩效评价指标体系}

\section{2 模糊计算过程}

（1）层次分析法计算指标权重

根据建设项目绩效评价指标体系，建立 $\mathrm{B}$ 层各元素相对于评价总目标 $\mathrm{A}$ 的两两判断矩阵。如 表 3 所示。

表3 A-B层的两两判断矩阵

\begin{tabular}{|c|c|c|c|c|c|}
\hline A & B1 & B2 & B3 & B4 & Wi \\
\hline B1 & 1.00 & 1.00 & 0.50 & 2.00 & 0.248 \\
\hline B2 & 1.00 & 1.00 & 2.00 & 0.50 & 0.248 \\
\hline B3 & 2.00 & 0.50 & 1.00 & 2.00 & 0.295 \\
\hline B4 & 0.50 & 2.00 & 0.50 & 1.00 & 0.209 \\
\hline
\end{tabular}

根据 1.1 层次分析法的步骤, 计算得到 $\lambda \max =4.57, \mathrm{CI}=0.19, \mathrm{RI}=0.89, \mathrm{CR}=0.21$ 因为 $\mathrm{CR}=0.21<0.10$, 这表明此两两判断矩阵满足一致性要求。

同时建立 A-B 层的两两判断矩阵相似，B-C 层两两判断矩阵及相应的 CR 如表 4-7 所示。 
表4投入

\begin{tabular}{|c|c|c|c|c|c|c|c|}
\hline $\mathrm{B} 1$ & $\mathrm{C} 11$ & $\mathrm{C} 12$ & $\mathrm{C} 13$ & $\mathrm{C} 14$ & $\mathrm{C} 15$ & $\mathrm{C} 16$ & $\mathrm{Wi}$ \\
\hline $\mathrm{C} 11$ & 1.00 & 0.50 & 2.00 & 1.00 & 1.00 & 2.00 & 0.185 \\
\hline $\mathrm{C} 12$ & 2.00 & 1.00 & 0.50 & 2.00 & 2.00 & 1.00 & 0.208 \\
\hline $\mathrm{C} 13$ & 0.50 & 2.00 & 1.00 & 0.50 & 0.50 & 2.00 & 0.147 \\
\hline $\mathrm{C} 14$ & 1.00 & 0.50 & 2.00 & 1.00 & 1.00 & 1.00 & 0.165 \\
\hline $\mathrm{C} 15$ & 1.00 & 0.50 & 2.00 & 1.00 & 1.00 & 1.00 & 0.165 \\
\hline $\mathrm{C} 16$ & 0.50 & 1.00 & 0.50 & 1.00 & 1.00 & 1.00 & 0.131 \\
\hline \multicolumn{7}{|c|}{$\lambda_{\max }=6.61$} \\
\hline
\end{tabular}

表5过程

\begin{tabular}{|c|c|c|c|c|c|c|c|c|}
\hline $\mathrm{B} 2$ & $\mathrm{C} 21$ & $\mathrm{C} 22$ & $\mathrm{C} 23$ & $\mathrm{C} 24$ & $\mathrm{C} 25$ & $\mathrm{C} 26$ & $\mathrm{C} 27$ & $\mathrm{Wi}$ \\
\hline $\mathrm{C} 21$ & 1.00 & 1.00 & 0.50 & 0.50 & 1.00 & 1.00 & 1.00 & 0.112 \\
\hline $\mathrm{C} 22$ & 1.00 & 1.00 & 1.00 & 1.00 & 2.00 & 2.00 & 2.00 & 0.184 \\
\hline $\mathrm{C} 23$ & 2.00 & 1.00 & 1.00 & 1.00 & 2.00 & 1.00 & 2.00 & 0.184 \\
\hline $\mathrm{C} 24$ & 2.00 & 1.00 & 1.00 & 1.00 & 2.00 & 1.00 & 2.00 & 0.184 \\
\hline $\mathrm{C} 25$ & 1.00 & 0.50 & 0.50 & 0.50 & 1.00 & 1.00 & 1.00 & 0.101 \\
\hline $\mathrm{C} 26$ & 1.00 & 0.50 & 1.00 & 1.00 & 1.00 & 1.00 & 1.00 & 0.124 \\
\hline
\end{tabular}

续表5

\begin{tabular}{|c|l|l|l|l|l|l|l|c|}
\hline $\mathrm{B} 2$ & $\mathrm{C} 21$ & $\mathrm{C} 22$ & $\mathrm{C} 23$ & $\mathrm{C} 24$ & $\mathrm{C} 25$ & $\mathrm{C} 26$ & $\mathrm{C} 27$ & $\mathrm{Wi}$ \\
\hline $\mathrm{C} 27$ & 1.00 & 0.50 & 0.50 & 0.50 & 1.00 & 1.00 & 1.00 & 0.112 \\
\hline \multicolumn{8}{|c|}{$\lambda_{\max }=7.14$} & $\mathrm{CI}=0.23 \mathrm{RI}=1.26 \quad \mathrm{CR}=0.18$ \\
\hline
\end{tabular}

表6 产出

\begin{tabular}{|c|c|c|c|c|c|c|}
\hline B3 & C31 & C32 & C33 & C34 & C35 & Wi \\
\hline C31 & 1.00 & 2.00 & 2.00 & 2.00 & 3.00 & 0.254 \\
\hline C32 & 0.50 & 1.00 & 1.00 & 2.00 & 2.00 & 0.221 \\
\hline C33 & 0.50 & 1.00 & 0.50 & 1.00 & 2.00 & 0.221 \\
\hline C34 & 0.50 & 0.50 & 1.00 & 1.00 & 2.00 & 0.193 \\
\hline C35 & 0.33 & 0.50 & 0.50 & 0.50 & 1.00 & 0.111 \\
\hline \multicolumn{7}{|c|}{$\lambda \lambda_{\max }=5.06 \quad \mathrm{CI}=0.02 \quad \mathrm{RI}=1.12 \quad \mathrm{CR}=0.01$} \\
\hline
\end{tabular}

表7 效果

\begin{tabular}{|c|c|c|c|c|c|c|}
\hline $\mathrm{B} 4$ & $\mathrm{C} 41$ & $\mathrm{C} 42$ & $\mathrm{C} 43$ & $\mathrm{C} 44$ & $\mathrm{C} 45$ & $\mathrm{Wi}$ \\
\hline $\mathrm{C} 41$ & 1.00 & 2.00 & 1.00 & 1.00 & 0.50 & 0.196 \\
\hline $\mathrm{C} 42$ & 0.50 & 1.00 & 2.00 & 0.50 & 1.00 & 0.171 \\
\hline $\mathrm{C} 43$ & 1.00 & 0.50 & 1.00 & 1.00 & 0.50 & 0.149 \\
\hline $\mathrm{C} 44$ & 1.00 & 2.00 & 1.00 & 1.00 & 2.00 & 0.259 \\
\hline $\mathrm{C} 45$ & 2.00 & 1.00 & 2.00 & 0.50 & 1.00 & 0.225 \\
\hline \multicolumn{7}{|c|}{$\lambda \max =5.39 \quad \mathrm{CI}=0.100 \quad \mathrm{RI}=1.12 \quad \mathrm{CR}=0.09$} \\
\hline
\end{tabular}

由表 4-7 可知, C 层各元素相对于各分目标 B 的两两判断矩阵中, 所有一致性比率 CR 都符合 一致性检验性标准。并由此可以得出所有评价指标的权重组合，如表 8 所示

(2) 制定评价指标 $C_{\mathrm{ij}}$ 的评分等级与评分标准

1) 确定评分等级

邀请由 20 位业内权威专家对该建设项目项目进行评价。在具体评价过程中, 由各专家根据指 标的含义和评估中的具体要求结合图 4.1 中所列各评价指标进行打分。在评价过程中各评价 指标的评价等级分为优、良、中、差、很差 5 级。其评分值分别为 $9 、 7 、 5 、 3 、 1 。$ 即本次 评价采用 10 分制法。 
2）确立专家打分法评分标准

评分标准如表 9 所示。

3 ) 获取评价指标评语集

由 20 位专家依据表 9 的评价标准对该桥梁项目的具体实施情况进行打分。例如, 对项目立项 规范性指标进行评价, 有 12 个人认为属于优, 6 个人认为属于良, 2 个人认为属于中。则可 以得出项目立项规范性的模糊评价矩阵为 $\left[\begin{array}{lllll}0.6 & 0.3 & 0.1 & 0 & 0\end{array}\right]$ 。所有评价指标的评语集如表 10 所 示。

表8 建设项目绩效评价各评价指标的相对权重

\begin{tabular}{|c|c|c|c|}
\hline B层目标 & 目标权重 & C层目标 & 目标权重 \\
\hline \multirow{6}{*}{ 投入 } & \multirow{6}{*}{0.248} & 项目立项规范性 & 0.185 \\
\hline & & 绩效目标合理性 & 0.208 \\
\hline & & 绩效指标明确性 & 0.147 \\
\hline & & 实施准备情况 & 0.165 \\
\hline & & 资金筹集 & 0.165 \\
\hline & & 资金落实 & 0.131 \\
\hline \multirow{7}{*}{ 过程 } & \multirow{7}{*}{0.248} & 制度执行有效性 & 0.112 \\
\hline & & 合同管理可控性 & 0.184 \\
\hline & & 项目质量可控性 & 0.184 \\
\hline & & 项目安全可控性 & 0.184 \\
\hline & & 管理制度健全性 & 0.101 \\
\hline & & 资金使用合规性 & 0.124 \\
\hline & & 财务监控有效性 & 0.112 \\
\hline \multirow{5}{*}{ 产出 } & \multirow{5}{*}{0.295} & 质量目标实现程度 & 0.254 \\
\hline & & 时间目标实现程度 & 0.221 \\
\hline & & 投资目标实现程度 & 0.221 \\
\hline & & 安全消防卫生目标实现程度 & 0.193 \\
\hline & & 节能目标实现程度 & 0.111 \\
\hline \multirow{5}{*}{ 效果 } & \multirow{5}{*}{0.209} & 经济效果 & 0.196 \\
\hline & & 社会效益 & 0.171 \\
\hline & & 生态效益 & 0.149 \\
\hline & & 可持续影响 & 0.259 \\
\hline & & 服务对象满意度 & 0.225 \\
\hline
\end{tabular}

(3) 一级综合评价

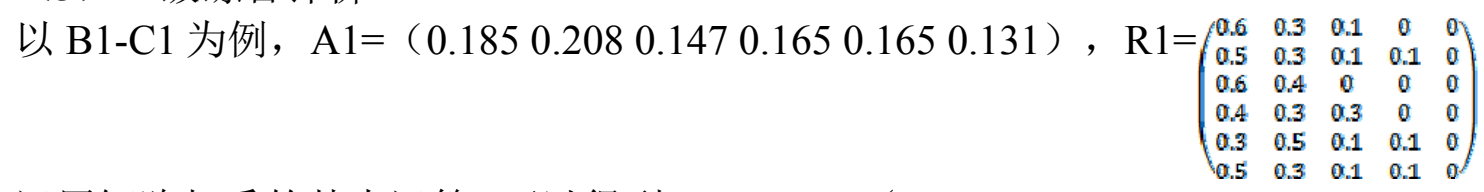

运用矩阵相乘的基本运算, 可以得到 $B 1=A 1 \circ R 1=\left(\begin{array}{lllll}0.4842 & 0.3850 & 0.1554 & 0.0504 & 0.0000\end{array}\right)$ 。同理, 可得 $B 2-C 2, B 3-C 3, B 4-C 4$ 的运算结果, 汇总结果如表 11 所示。

(4) 二级综合评价

本级综合评价是 A-B 级模糊综合评价, 首先构造权重向量 $\mathrm{A}$, 即 B 层指标对 A 层指标的权重 [6]。 $\mathrm{A}=\left(\begin{array}{l}0.2480 .2480 .2950 .209\end{array}\right)$

模糊关系矩阵 $\mathrm{R}=(\mathrm{B} 1 \mathrm{~B} 2 \mathrm{~B} 3 \mathrm{~B} 4)=\left(\begin{array}{ccccc}0.4842 & 0.3850 & 0.1554 & 0.0504 & 0.0000 \\ 0.5193 & 0.2803 & 0.1286 & 0.0472 & 0.0248 \\ 0.4075 & 0.3077 & 0.2627 & 0.0000 & 0.0000 \\ 0.6269 & 0.2633 & 0.0902 & 0.0196 & 0.0000\end{array}\right)$ 


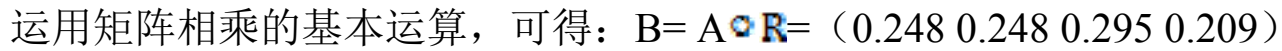
0.4842
0.5193
0.4075
0.6269
0.1554
0.0504
0.0000
$0.2803 \quad 0.1286$
$0.3077 \quad 0.2627$
0.0472
0.0000
0.0248
( 0.49760 .30970 .16640 .02820 .0062$)$

根据二级综合评价的结果，可以得出结论：对于该桥梁项目绩效评价，有 $49.76 \%$ 的专家认为 评价结果属于优, 有 $30.97 \%$ 的专家认为评价结果为良。有 $16.64 \%$ 的专家认为评价结果为中, 还有少量专家认为评价结果为差或者很差。取所有专家的众数作为本次绩效评价的最终结论, 即该桥梁项目绩效评价结论为优。

\section{表9 各评价指标的评价标准}

\begin{tabular}{|c|c|c|c|}
\hline $\begin{array}{l}\text { A层指 } \\
\text { 标 }\end{array}$ & $\begin{array}{c}\mathrm{B} \text { 层目 } \\
\text { 标 }\end{array}$ & C层目标 & 评价标准 \\
\hline \multirow{23}{*}{$\begin{array}{l}\text { 某桥梁 } \\
\text { 项目绩 } \\
\text { 效评价 }\end{array}$} & \multirow{6}{*}{ 投入 } & $\begin{array}{c}\text { 项目立项规范 } \\
\text { 性 }\end{array}$ & $\begin{aligned} \text { 项目决策实际所用时间短, 各类手续齐全、审批及时, 按照规定的 } \\
\text { 程序申请设立 }\end{aligned}$ \\
\hline & & $\begin{array}{l}\text { 绩效目标合理 } \\
\text { 性 }\end{array}$ & 项目目标明确化、系统化、且大部分目标可测量, 便于目标的控制 \\
\hline & & $\begin{array}{l}\text { 绩效指标明确 } \\
\text { 性 }\end{array}$ & 项目质量、投资、劳动安全卫生、环境等目标明确 \\
\hline & & 实施准备情况 & 项目勘察、设计、招投标组织实施等完成程度 \\
\hline & & 资金筹集 & 资金审核合规 \\
\hline & & 资金落实 & 资金到位合理、按项目进度及时到位 \\
\hline & \multirow{7}{*}{ 过程 } & $\begin{array}{c}\text { 制度执行有效 } \\
\text { 性 }\end{array}$ & $\begin{array}{c}\text { 制定的业务管理制度、建设管理组织机构科学合理, 控制手段严谨 } \\
\text { 有效 }\end{array}$ \\
\hline & & $\begin{array}{l}\text { 合同管理可控 } \\
\text { 性 }\end{array}$ & 合同变更程序规范、项目有效应对变更 \\
\hline & & $\begin{array}{c}\text { 项目质量可控 } \\
\text { 性 }\end{array}$ & 工程质量达到法律法规的规定, 满足项目建设需求 \\
\hline & & $\begin{array}{l}\text { 项目安全可控 } \\
\text { 性 }\end{array}$ & 项目安全措施明确可行, 无重大伤亡事故 \\
\hline & & $\begin{array}{l}\text { 管理制度健全 } \\
\text { 性 }\end{array}$ & 资金管理安全透明、会计核算规范, 跟踪审计核减率合理 \\
\hline & & $\begin{array}{c}\text { 资金使用合规 } \\
\text { 性 }\end{array}$ & 没有超概现象，资金利用率合理 \\
\hline & & $\begin{array}{l}\text { 财务监控有效 } \\
\text { 性 }\end{array}$ & $\begin{array}{c}\text { 财务监控措施透明，制度建设健全，审查符合有关规定，没有资金 } \\
\text { 违纪现象 }\end{array}$ \\
\hline & \multirow{5}{*}{ 产出 } & $\begin{array}{l}\text { 质量目标实现 } \\
\text { 程度 }\end{array}$ & 工程质量达到法律法规的规定, 满足项目建设需求 \\
\hline & & $\begin{array}{l}\text { 时间目标实现 } \\
\text { 程度 }\end{array}$ & 按工期完工 \\
\hline & & $\begin{array}{l}\text { 投资目标实现 } \\
\text { 程度 }\end{array}$ & 将成本控制在概算内 \\
\hline & & $\begin{array}{l}\text { 安全消防卫生 } \\
\text { 目标实现程度 }\end{array}$ & 劳动安全卫生消防目标满足项目需求 \\
\hline & & $\begin{array}{l}\text { 节能目标实现 } \\
\text { 程度 }\end{array}$ & 资源消耗合理性降低 \\
\hline & \multirow{5}{*}{ 效果 } & 经济效果 & 投资回收期、效益费用比等合理 \\
\hline & & 社会效益 & 增强道路服务能力, 缩短行车时间 \\
\hline & & 生态效益 & “三废”、噪声污染及水土流失、植被破坏程度全部控制在标准之内 \\
\hline & & 可持续影响 & 对项目团队和沿线经济的可持续影响 \\
\hline & & $\begin{array}{c}\text { 服务对象满意 } \\
\text { 度 }\end{array}$ & 沿线居民及行车人员满意度高 \\
\hline
\end{tabular}


表10 所有评价指标的模糊综合评价矩阵

\begin{tabular}{|c|c|c|c|c|c|c|}
\hline \multirow{2}{*}{ 准则层B } & \multirow{2}{*}{ 指标层C } & \multicolumn{5}{|c|}{ 模糊综合评价矩阵 } \\
\hline & & 优 & 良 & 中 & 差 & 很差 \\
\hline \multirow{6}{*}{ 投入 } & 项目立项规范性 & 0.6 & 0.3 & 0.1 & 0 & 0 \\
\hline & 绩效目标合理性 & 0.5 & 0.3 & 0.1 & 0.1 & 0 \\
\hline & 绩效指标明确性 & 0.6 & 0.4 & 0 & 0 & 0 \\
\hline & 实施准备情况 & 0.4 & 0.3 & 0.3 & 0 & 0 \\
\hline & 资金筹集 & 0.3 & 0.5 & 0.1 & 0.1 & 0 \\
\hline & 资金落实 & 0.5 & 0.3 & 0.1 & 0.1 & 0 \\
\hline \multirow{5}{*}{ 过程 } & 制度执行有效性 & 0.2 & 0.5 & 0.3 & 0 & 0 \\
\hline & 合同管理可控性 & 0.8 & 0.2 & 0 & 0 & 0 \\
\hline & 项目质量可控性 & 0.7 & 0.2 & 0.1 & 0 & 0 \\
\hline & 项目安全可控性 & 0.5 & 0.4 & 0.1 & 0 & 0 \\
\hline & 管理制度健全性 & 0.7 & 0.3 & 0 & 0 & 0 \\
\hline \multirow{2}{*}{ 准则层B } & \multirow{2}{*}{ 指标层C } & \multicolumn{5}{|c|}{ 模糊综合评价矩阵 } \\
\hline & & 优 & 良 & 中 & 差 & 很差 \\
\hline \multirow{2}{*}{ 过程 } & 资金使用合规性 & 0.2 & 0.2 & 0.2 & 0.2 & 0.2 \\
\hline & 财务监控有效性 & 0.3 & 0.2 & 0.3 & 0.2 & 0 \\
\hline \multirow{5}{*}{ 产出 } & 质量目标实现程度 & 0.8 & 0.2 & 0 & 0 & 0 \\
\hline & 时间目标实现程度 & 0.1 & 0.5 & 0.3 & 0.1 & 0 \\
\hline & 投资目标实现程度 & 0.2 & 0.3 & 0.5 & 0 & 0 \\
\hline & 安全消防卫生目标实现程度 & 0.6 & 0.3 & 0.1 & 0 & 0 \\
\hline & 节能目标实现程度 & 0.2 & 0.2 & 0.6 & 0 & 0 \\
\hline \multirow{5}{*}{ 效果 } & 经济效果 & 0.5 & 0.3 & 0.1 & 0.1 & 0 \\
\hline & 社会效益 & 0.5 & 0.5 & 0 & 0 & 0 \\
\hline & 生态效益 & 0.4 & 0.3 & 0.3 & 0 & 0 \\
\hline & 可持续影响 & 0.7 & 0.2 & 0.1 & 0 & 0 \\
\hline & 服务对象满意度 & 0.9 & 0.1 & 0 & 0 & 0 \\
\hline
\end{tabular}

表11 一级综合评价汇总结果

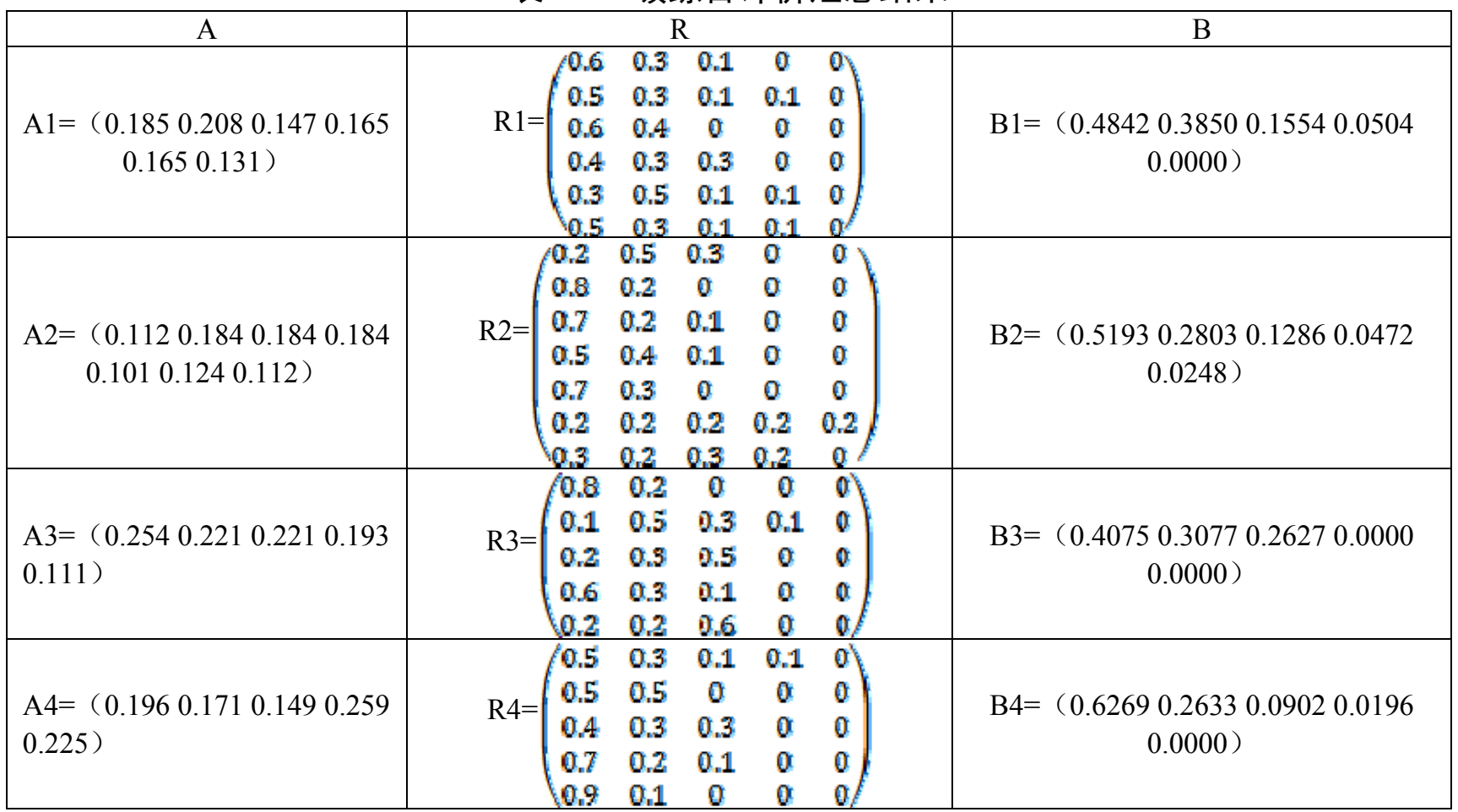




\section{AHP-模糊综合评价法在建设项目绩效评价应用中的评价}

\section{1 此方法应用的可行性}

(1) 对于难以给出是或者不是概念的绩效评价工作, 可以采取层次分析法与模糊综合评估法 双法结合的方式将这一工作进行量化计算，以得到更加准确有效的评价结果 ${ }^{[7]}$ 。

（2）如果未来的建设项目绩效评价在AHP-模糊综合评价的基础上进一步借助计算机进行初 始数据的分析与计算, 则可以快速、便捷地得出项目绩效评价的最终结论。

5.2 此方法应用的局限性

将此方法应用到绩效评价工作时, 需两次对定性部分进行打分, 虽然与纯粹定性分析相比有 了较高的可信度, 但如果专家数量不足, 即原始数据不准确, 则依然可能出现评价结果出现 差错的情况

\section{References}

[1]Jianzhong Wang. Construction project management guidelines. Tianjin University Press, 2013, p. 365.

[2]Lili Wang. Research on Fuzzy mathematics method combined with analytic hierarchy process using for cleaner production potential evaluation, master, Chongqing University, China, 2010.

[3]Yuhua Wu, Gang Du. Foundations of Management Science. Tianjin University Press, 2009, p.235.

[4]Yu Liu. Research on the fuzzy comprehensive evaluation method of urban land intensive utilization evaluation, master, Nanjing Normal University, 2008.

[5]Zheng Zhang, Tianbiao Yu, et al. Research on supplier evaluation by analytic hierarchy process and fuzzy comprehensive evaluation. Journal of Northeastern University, 2006, No. 10, p. $1142-1145$.

[6]Lina Zhang. AHP- The application of ecological industrial park by fuzzy comprehensive evaluation method .master, Dalian University of Technology, 2006.

[7]Lixiang Duan, Laibin Zhang. AHP- the application in centrifugal pump of safety evaluation by Fuzzy mathematics method. Journal of safety science and technology, Vol 07(2011) No. 02.p. 127-131. 